\title{
Synthesis of Chiral Tertiary Boronic Esters: Phosphonate-Directed Catalytic Asymmetric Hydroboration of Trisubstituted Alkenes
}

\author{
Suman Chakrabarty ${ }^{\circledR}$ and James M. Takacs*우 \\ Department of Chemistry, University of Nebraska-Lincoln, Lincoln, Nebraska 68588-0304, United States
}

Supporting Information

ABSTRACT: Highly enantioselective rhodium-catalyzed hydroboration of allylic phosphonates by pinacolborane affords chiral tertiary boronic esters. The $\beta$-borylated phosphonates are readily converted to chiral $\beta$ - and $\gamma$ hydroxyphosphonates and aminophosphonates and to phosphonates bearing a quaternary carbon stereocenter. The utility of the latter is illustrated by the synthesis of (S)-(+)-bakuchiol methyl ether.

$\mathrm{C}$ hiral organoboronates are valuable reagents in asymmetric synthesis due to the versatility with which the C$\mathrm{B}$ bond can be utilized via a myriad of diverse stereospecific transformations. ${ }^{1}$ The direct introduction of boron via the catalytic asymmetric hydroboration (CAHB) of alkenes, ${ }^{2}$ particularly of vinylarenes, ${ }^{3}$ has received much recent interest. ${ }^{4,5}$ We focus on accessing functionalized, chiral boronic esters via rhodium-catalyzed, directed $\mathrm{CAHB}$ of $\beta, \gamma$-unsaturated substrates. ${ }^{6}$ For example, the oxime-directed $\mathrm{CAHB}$ of trisubstituted alkene (E)-1 affords the novel chiral, tertiary boronic ester $(R)-2$ (84\%, 95:5 er; Figure 1). ${ }^{6 a}$ The TADDOL-derived chiral cyclic monophosphite ligand $(R, R)$ T1 is used to control the $\pi$-facial selectivity.

\section{Prior Work: Oxime-directed CAHB}<smiles>CC(C)=NOC/C(C)=C\CCc1ccccc1</smiles>

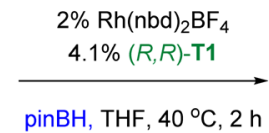

$(E)-1$

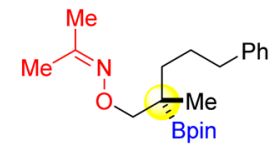

(R)-2 (84\%, 95:5 er)
Current Work: Phosphonate-directed CAHB<smiles>CCOP(=O)(OCC)/C(C)=C/CCc1ccccc1</smiles>

$(E)-3 a$

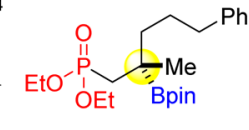

$(R)-4 a(82 \%, 99: 1$ er $)$

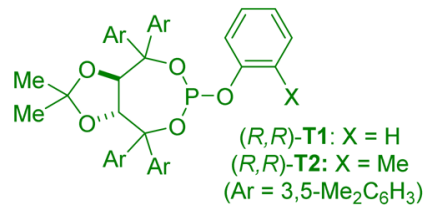

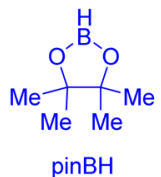

\section{$0.25 \%[\mathrm{Rh}(\operatorname{cod}) \mathrm{Cl}]_{2}, 0.5 \% \mathrm{AgBF}_{4}$ $0.5 \%(R, R)-\mathrm{T} 2$ (in situ generated precatalyst) $\operatorname{pinBH}, T H F, r t, 2$ h}

Figure 1. Chiral tertiary boronic esters via phosphonate-directed CAHB ( $\mathrm{nbd}=$ norbornadiene cod $=$ cyclooctadiene $)$.
Encouraged by the success of oxime-directed CAHB, we turned our attention to exploring the potential effectiveness of phosphonate functionality as a directing group. Recently, phosphonates have been elegantly used to enable novel modes of $\mathrm{C}-\mathrm{H}$ activation. ${ }^{7}$ However, the overall effectiveness of phosphonates as directing groups in asymmetric catalysis remains largely unexplored. We now report that phosphonate (E)-3a undergoes efficient borylation to yield the chiral tertiary boronic ester $4 \mathbf{a}$ in excellent yield (82\%) and with high levels of enantioinduction (99:1 er). While commercial $\mathrm{Rh}(\mathrm{nbd})_{2} \mathrm{BF}_{4}$ is a suitable catalyst precursor, a catalyst generated in situ from $[\mathrm{Rh}(\operatorname{cod}) \mathrm{Cl}]_{2}$ and $\mathrm{AgBF}_{4}$, in combination with the TADDOLderived chiral monophosphite $\mathbf{T} 2{ }^{8}$ is more economical and efficiently catalyzes hydroboration with pinacolborane (pinBH). We often find that the nature of the directing group strikingly influences the regio- and stereoselectivity of the CAHB. ${ }^{6}$ In the present case, however, the observed $\beta$ regiochemistry and $\pi$-facial selectivity for CAHB of (E)-3a match those of oxime-directed borylation (Figure 1).

Chiral phosphonates, particularly hydroxy- and aminophosphonates are bioisosteres of the corresponding amino acids and key structural elements of antibiotics, antiviral, and anticancer drugs. ${ }^{9}$ However, the toolbox for introducing chirality via the functionalization of phosphonates is largely limited to catalytic asymmetric hydrogenation. ${ }^{10,11}$ Chiral, borylated phosphonates can enable new possibilities as chiral synthons. Figure 2 illustrates stereospecific transformations we have investigated for tertiary boronic ester $\mathbf{4 a}$, including its conversion to chiral $\beta$ - and $\gamma$-hydroxyphosphonates and aminophosphonates and to phosphonates bearing a quaternary all-carbon stereocenter. All-carbon quaternary stereocenters are a common structural motif in bioactive natural products and pharmaceutical drugs. ${ }^{12}$

The reaction of $(E)$-3a has been carried out on gram scale using a $0.5 \mathrm{~mol} \%$ catalyst loading and only a slight excess of pinBH (1.1 equiv). Oxidation of $4 a$ by $\mathrm{NaBO}_{3}$ yields the chiral, tertiary $\beta$-hydroxyphosphonate 5a (95\%, 99:1 er). Cross-couplings of $\mathbf{4 a}$ under conditions reported by Aggarwal et al. are facile and afford the furan derivative $7 \mathbf{a}(71 \%)^{1 \mathrm{e}}$ and the vinylated derivative 8a $(93 \%){ }^{13}$ Attempts to convert $4 \mathbf{4 a}$ directly to latent aldehyde or carboxylic acid moieties using the typical conditions employed for boronic esters ${ }^{13}$ were not successful, perhaps due to the all-alkyl substitution pattern of the tertiary boronic ester in $\mathbf{4 a}$. However, ozonolysis of $\mathbf{8 a}$ followed by a mild reductive workup ${ }^{14}$ yields the chiral

Received: March 11, 2017

Published: April 17, 2017 

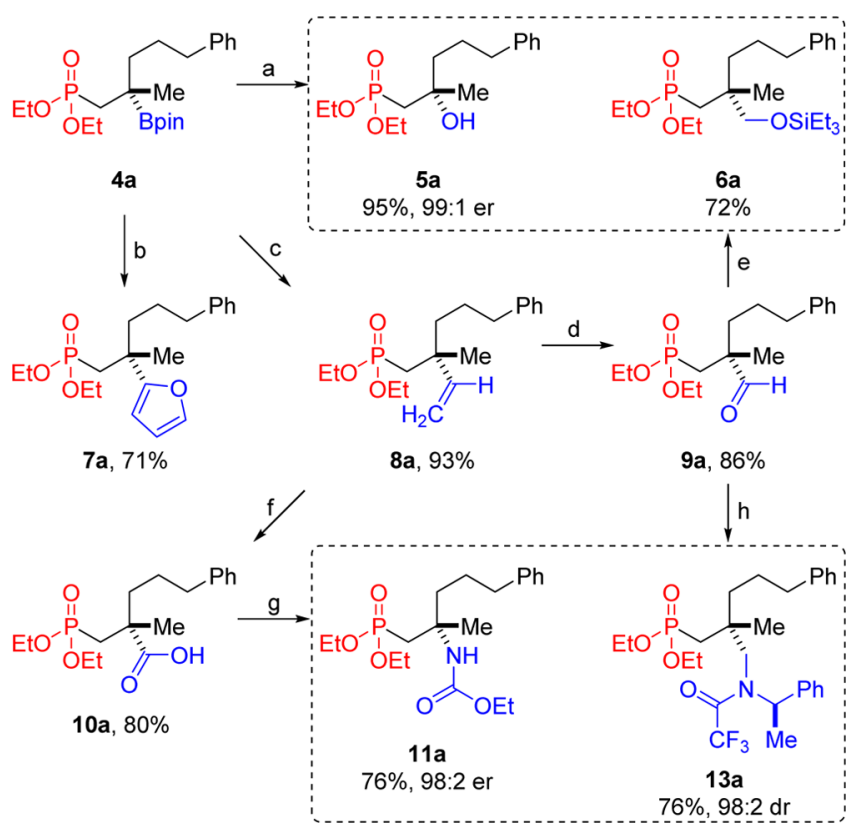

Figure 2. Utility of phosphonate functionalized tertiary boronic esters is illustrated by selected transformations of $4 \mathbf{a}$. Reagents and conditions: (a) $\mathrm{NaBO}_{3} \cdot 4 \mathrm{H}_{2} \mathrm{O}$; (b) (i) $n \mathrm{BuLi}$, furan, $-78^{\circ} \mathrm{C}$, THF; (ii) NBS; (iii) aq. $\mathrm{Na}_{2} \mathrm{~S}_{2} \mathrm{O}_{3}$; (c) (i) $\mathrm{CH}_{2}=\mathrm{CHMgBr}$, THF; (ii) $\mathrm{I}_{2}$, $\mathrm{MeOH}$ (iii) $\mathrm{MeONa}, \mathrm{MeOH}$; (d) (i) $\mathrm{O}_{3}, \mathrm{CH}_{2} \mathrm{Cl}_{2}, 0{ }^{\circ} \mathrm{C}$; (ii) $\mathrm{Et}_{3} \mathrm{~N}$, rt (e) $\mathrm{Et}_{3} \mathrm{SiH},\left[\mathrm{Ru}(p \text {-cymene }) \mathrm{Cl}_{2}\right]_{2}$, toluene, $50{ }^{\circ} \mathrm{C}$; (f) (i) $\mathrm{O}_{3}, \mathrm{CH}_{2} \mathrm{Cl}_{2}$, $0{ }^{\circ} \mathrm{C}$; (ii) $\mathrm{Et}_{3} \mathrm{~N}$; (iii) $\mathrm{NaH}_{2} \mathrm{PO}_{4}, \mathrm{NaClO}_{2}$, 2-methyl-2-butene, $t$ - $\mathrm{BuOH}$, rt; (g) (i) DPPA, toluene reflux; (ii) EtOH; (h) (i) (R)-(+)- $\alpha$ methylbenzylamine, $\mathrm{AcOH}, \mathrm{NaCNBH}_{3}$; (ii) $\left(\mathrm{CF}_{3} \mathrm{CO}\right)_{2} \mathrm{O}, \mathrm{Et}_{3} \mathrm{~N}, \mathrm{THF}$, rt.

phosphonoaldehyde 9a (86\%). Ruthenium-catalyzed reductive silylation $^{15}$ affords the silyl protected chiral $\gamma$-hydroxyphosph- onate $6 \mathbf{a}(72 \%)$. Reductive amination ${ }^{16}$ of $9 \mathbf{a}$ with $(R)-(+)-\alpha$ methylbenzylamine yields 12a (see the Supporting Information (SI)) which followed by acylation affords the chiral $\gamma$ aminophosphonate 13a (76\% overall, 98:2 dr). Our attempts to convert $4 \mathbf{a}$ directly to the chiral, tertiary $\beta$-aminophosphonate $^{10}$ using several commonly employed methods ${ }^{17}$ were unsuccessful. However, ozonolysis of $\mathbf{8 a}$ followed by Pinnick oxidation ${ }^{3 \mathrm{~b}}$ affords the chiral carboxylic acid 10a (80\%). Its conversion to the chiral $\beta$-aminophosphonate 11a (76\%, 98:2 er) via Curtius rearrangement proceeded smoothly under standard conditions. ${ }^{18}$

Figure 3 summarizes results obtained for a series of trisubstituted alkenes differing in the nature of alkyl chain at the position labeled $R^{E}$. Substrates similar to $3 \mathbf{a}$ bearing substituted aromatics and heteroaromatics (i.e., $3 \mathbf{b}-\mathbf{e}$ ) undergo efficient $\beta$-borylation. For example, the trifluoromethylphenyl derivative $\mathbf{3 b}$ affords $\mathbf{4 b}(78 \%$, >99:1 er). The 4chlorophenyl derivative $4 \mathrm{c}(76 \%, 99: 1 \mathrm{er})$ could in principle be used in subsequent cross-coupling chemistry, further highlighting the goal to prepare multifunctional synthons via CAHB. Substrates $\mathbf{3 d}$ and $\mathbf{3 e}$ demonstrate that simple heteroaromatic ring systems can be carried through the CAHB sequence; 4d (77\%, 98.5:1.5 er) and 4e (71\%, 97:3 er) are obtained in good yields and high enantioselectivities.

Substrates with saturated alkyl substituents (i.e., $\mathbf{3 f}$ and $\mathbf{3 g}$ ) give $\mathbf{4 f}(83 \%, 97: 3 \mathrm{er})$ and $\mathbf{4 g}(80 \%, 98: 2 \mathrm{er})$, respectively. The structurally related chiral substrate $3 \mathrm{~h}$ undergoes highly diastereoselective CAHB ( $>20: 1 \mathrm{dr})$ with catalyst control; $(R, R)$-T2 affords $(R, S)-4 \mathbf{h}(83 \%)$, and $(S, S)$-T2 affords $(S, S)$ 4h $(82 \%)$. $\beta$-Borylated products bearing a Boc-protected nitrogen substituent $(4 \mathbf{i})$ or hydroxy substituents, protected as the benzoate, benzyl ether, or benzyloxymethyl ether (i.e., $4 \mathbf{j}-$ 1), are obtained with high levels of asymmetric induction. The chiral acetal substrate $3 \mathrm{~m}$ undergoes efficient CAHB, again

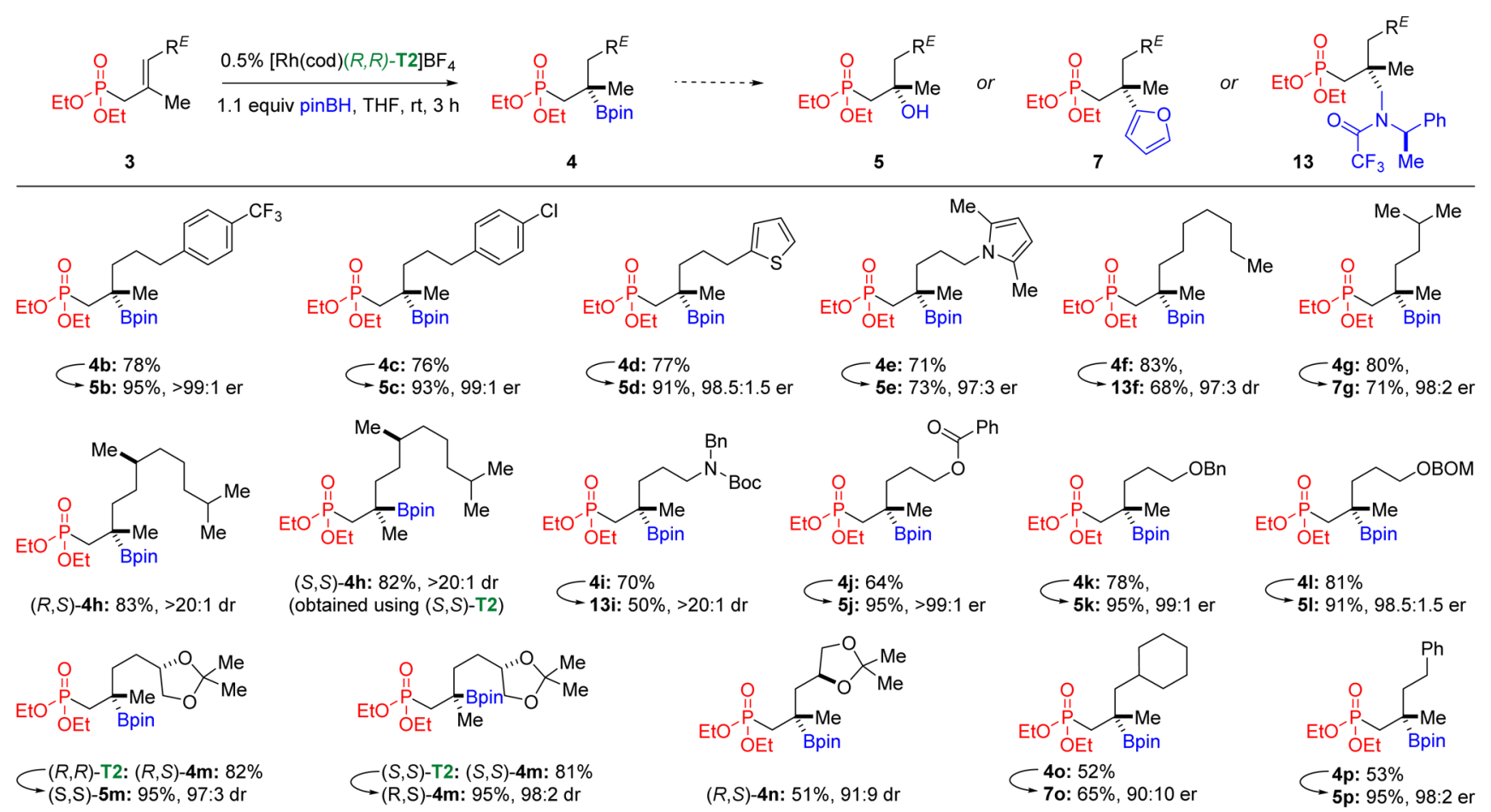
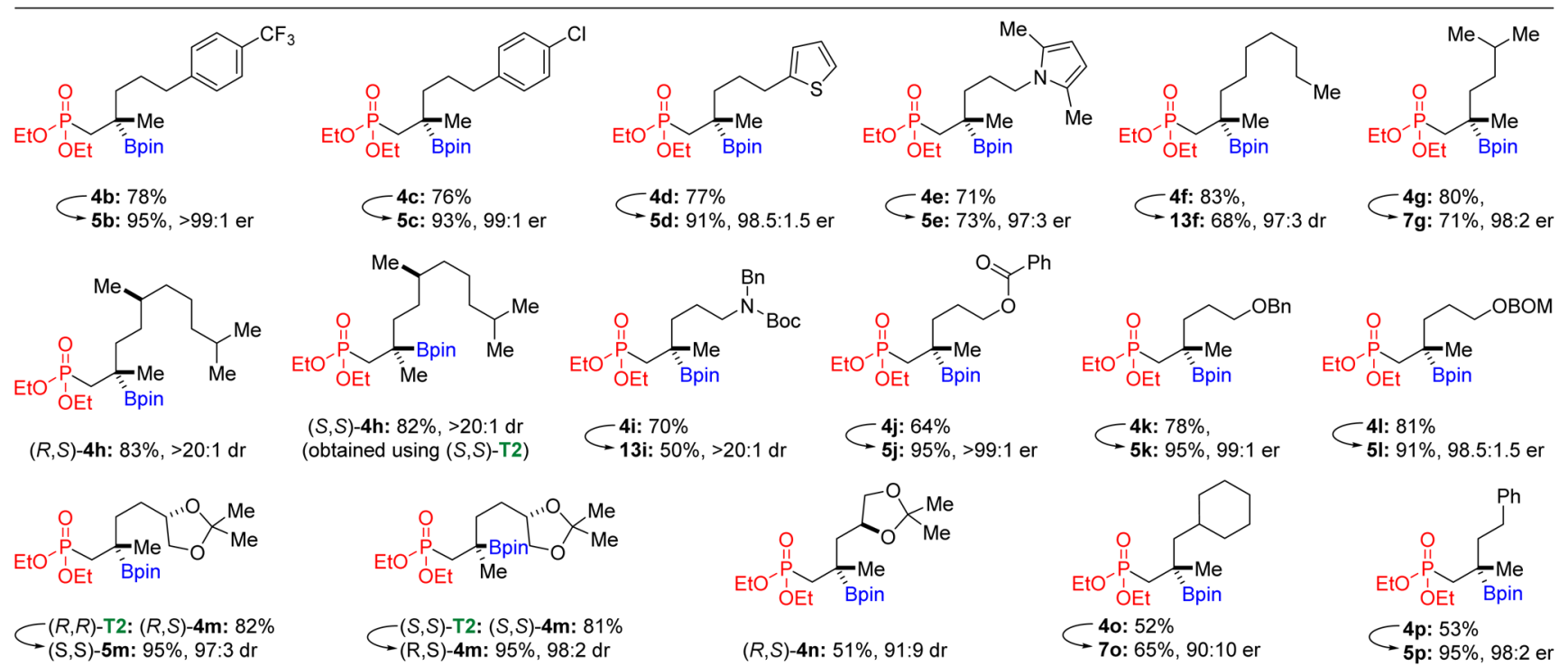

Figure 3. Substrate scope. Note: er is determined either by chiral HPLC analysis of tertiary alcohol derivative 5 or cross-coupled derivative 7 or by NMR analysis of the amino derivative 13. 
with good catalyst control over diastereoselectivity. Depending on the configuration of $\mathbf{T} 2$, either $(R, S)-\mathbf{4 m}$ or $(S, S)-\mathbf{4} \mathbf{m}$ is obtained in high yield $(81-82 \%)$ and high diastereoselectivity, 97:3 dr and 98:2 dr, respectively. The one carbon shorter analogue $3 \mathbf{n}$ affords $(R, S)-\mathbf{4 n}(51 \%, 91: 9 \mathrm{dr})$ using $(R, R)-\mathbf{T} 2$ but forms a complex mixture using $(S, S)$-T2. Substrates related to $3 \mathbf{n}$, bearing a bulkier vinyl substituent such as in $3 \mathbf{o}$ and $3 \mathbf{p}$, and substrates related to $3 \mathbf{a}$, in which the vinyl methyl substituent is replaced by bulkier substituents, tend to react more sluggishly and give more side products; for example, lower yields are obtained for 40 (52\%, 90:10 er) and $4 p$ (53\%, 98:2 er). The alkene geometry plays an important role in the reaction. (Z)-3a yields (R)-4a (40\%, 99:1 er) with high enantioselectivity but in much lower yield compared to $(E)$ 3a. ${ }^{19}$

In addition to their potential as pharmacophores for medicinal chemistry, phosphonates enable useful synthetic transformations. A formal total synthesis of the natural product (S)-(+)-bakuchiol ${ }^{20,21}$ further illustrates the synthetic utility of these chiral phosphonate-functionalized, tertiary boronic esters (Figure 4). (S)-(+)-Bakuchiol possesses a remote alkene as well as a challenging skipped diene subunit in which the two alkene moieties are separated by a quaternary all-carbon stereocenter. We envisioned utilizing the chemistry of the boronic ester and the phosphonate in $\mathbf{4 q}$ sequentially to form the skipped diene subunit.

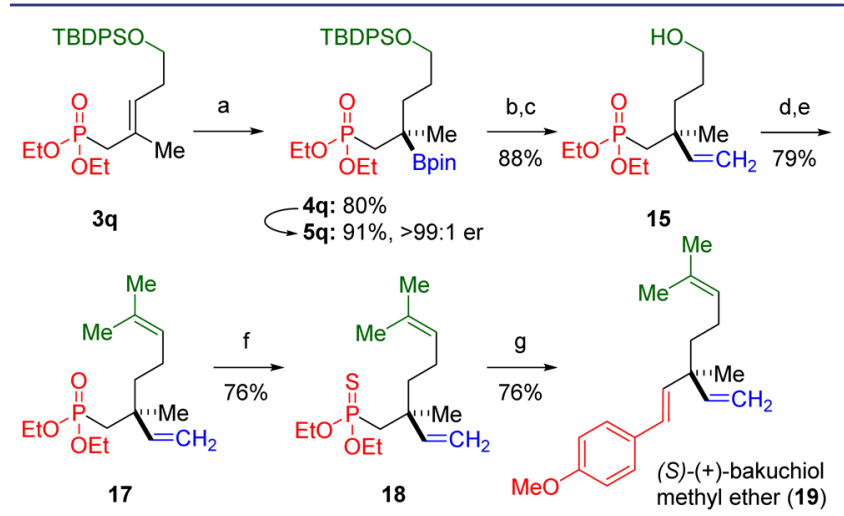

Figure 4. Formal total synthesis of $(S)-(+)$-bakuchiol. Reagents and conditions: (a) $0.25 \%[\mathrm{Rh}(\mathrm{cod}) \mathrm{Cl}]_{2}, 0.50 \% \mathrm{AgBF}_{4}, 0.50 \%(S, S)-\mathrm{T} 2$, 1.1 equiv of pinBH, THF $(c=1 \mathrm{M})$, rt, $12 \mathrm{~h}$; (b) (i) $\mathrm{CH}_{2}=\mathrm{CHMgBr}$, THF, $-78{ }^{\circ} \mathrm{C}$; (ii) $\mathrm{I}_{2}, \mathrm{MeOH},-78{ }^{\circ} \mathrm{C}$; (iii) $\mathrm{NaOMe}, \mathrm{MeOH}$; (iv) $\mathrm{Na}_{2} \mathrm{~S}_{2} \mathrm{O}_{3}$ (aq.); (c) TBAF, $\mathrm{H}_{2} \mathrm{O}$; (d) DMSO, $\mathrm{Py} \cdot \mathrm{SO}_{3}$, Hünig's base; (e) $\left(\mathrm{CH}_{3}\right)_{2} \mathrm{CH}=\mathrm{PPh}_{3}$; (f) Lawesson's reagent, toluene reflux; (g) $n \mathrm{BuLi}$, 4-methoxy-benzaldehyde.

CAHB of $\mathbf{3 q}$ with $(S, S)-\mathbf{T} \mathbf{2}$ affords $\mathbf{4 q}$ ( $80 \%$ on gram scale); the enantioselectivity $(>99: 1 \mathrm{er})$ is determined after oxidation to tertiary alcohol $\mathbf{5 q}$. Cross-coupling of $\mathbf{4 q}$ with vinyl magnesium bromide ${ }^{13}$ to 14 (see the SI) followed by deprotection of the silyl ether yields 15. Oxidation to the aldehyde 16 (see the SI) followed by Wittig olefination to 17 sets the stage for exploiting the phosphonate functionality to complete the synthesis. Direct phosphonate olefination is limited in scope since $\beta$-hydroxy phosphonates lacking electron withdrawing substituents on the $\alpha$-carbon are not prone to eliminate without activation. ${ }^{22}$ However, Corey $^{23}$ found that $\beta$ hydroxy thionophosphonates readily undergo elimination to form alkenes. Treating phosphonate 17 with Lawesson's reagent $^{24}$ affords thionophosphonate 18. Deprotonation by $n \mathrm{BuLi}$ followed by the addition of 4-methoxy benzaldehyde smoothly yields $(S)-(+)$-bakuchiol methyl ether $(19){ }^{25}$ Conversion of 19 to the natural product was previously reported. $^{21 a}$ Since either enantiomer of the chiral monophosphite T2 is equally accessible, a sequence beginning with $(R, R)$-T2 was carried out to give the enantiomeric $(R)$ (-)-bakuchiol methyl ether. ${ }^{21 \mathrm{c}}$

Figure 5 summarizes data obtained probing three mechanistic aspects of phosphonate-directed CAHB. (i) The distance separating the phosphonate directing group and the alkene undergoing reaction is a key factor for efficient reaction. While (E)-3a reacts efficiently to yield the boronic ester $4 \mathbf{a}$, the one carbon homologue $\mathbf{2 0}$ is unreactive under the same conditions. We speculate that efficient chelation by the substrate is necessary for efficient catalysis. (ii) The ligand-to-metal ratio (i.e., T2:Rh) strongly influences the activity of the catalyst. Graph A (Figure 5) compares the yield of $4 \mathbf{a}$ over time for catalysts prepared with a 1:1 T2:Rh ratio (blue line) and 2:1 T2:Rh ratio (red line). The catalyst formed using a 1:1 T2:Rh yields $4 a$ in about $80 \%$ yield after roughly an hour. The catalyst formed using a 2:1 $\mathbf{T} 2: \mathrm{Rh}$ ratio produces $4 \mathrm{a}$ but in only $60 \%$ yield after roughly $4 \mathrm{~h}$. While the reaction is much slower, the enantiomer ratio of $4 \mathrm{a}(99: 1 \mathrm{er})$ is unchanged by the change in T2:Rh ratio. (iii) Graph B (Figure 5) shows the linear dependence of percent ee of product $\mathbf{4 a}$ on the enantiomeric purity of $\mathbf{T} 2$. The lack of a nonlinear effect ${ }^{26}$ in this case is consistent with a 1:1 T2:Rh complex in the active catalyst.

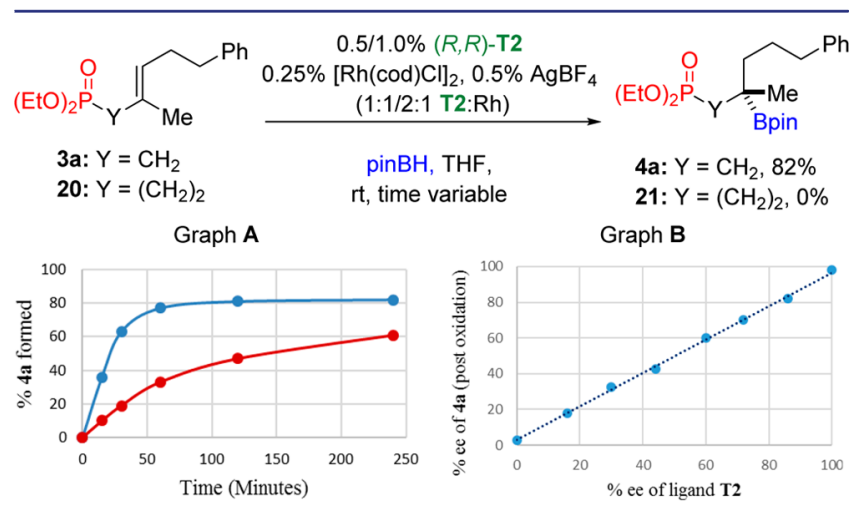

Figure 5. Key mechanistic considerations: Graph A compares the yield of $\mathbf{4 a}$ over time for catalysts prepared using 1:1 (blue line) versus 2:1 (red line) T2:Rh ratios; Graph B plots percent ee of the product versus percent ee of the chiral catalyst.

In conclusion, the successful use of phosphonate functionality as a directing group in Rh-catalyzed CAHB of trisubstituted alkenes leads to the formation of functionalized chiral, tertiary boronic esters, in high yield (up to 83\%) and with high levels of enantioselectivity (up to 99:1 er, or greater). A simple TADDOL-derived ligand system efficiently controls the $\pi$-facial selectivity, and the reaction exhibits tolerance toward a range of functional groups. Stereospecific routes to generate quaternary all-carbon stereocenters are demonstrated, and the multifunctional utility of these novel molecules as chiral synthons is demonstrated in the synthesis of bakuchiol. Mechanistic experiments indicate that a $1: 1 \mathrm{Rh}$ to-monophosphite complex is relevant to catalysis. Further studies are in progress. 


\section{ASSOCIATED CONTENT}

\section{S Supporting Information}

The Supporting Information is available free of charge on the ACS Publications website at DOI: 10.1021 /jacs.7b02324.

Experimental procedures and characterization data (PDF)

NMR data (PDF)

\section{AUTHOR INFORMATION}

\section{Corresponding Author}

*jtakacs1@unl.edu

\section{ORCID}

Suman Chakrabarty: 0000-0002-6611-3839

James M. Takacs: 0000-0002-1903-6535

Notes

The authors declare no competing financial interest.

\section{ACKNOWLEDGMENTS}

Funding from the NIH National Institutes of General Medical Sciences (R01-GM100101) is gratefully acknowledged. We sincerely thank P. H. Dussault (UNL) for use of equipment and V. M. Shoba (UNL) for helpful discussions.

\section{REFERENCES}

(1) Recent reviews: (a) Leonori, D.; Aggarwal, V. K. Angew. Chem., Int. Ed. 2015, 54, 1082. (b) Scott, H. K.; Aggarwal, V. K. Chem. - Eur. J. 2011, 17, 13124. Recent methodology developments: (c) Zhang, L.; Lovinger, G. J.; Edelstein, E. K.; Szymaniak, A. A.; Chierchia, M. P.; Morken, J. P. Science 2016, 351, 70. (d) Matthew, S. C.; Glasspoole, B. W.; Eisenberger, P.; Crudden, C. M. J. Am. Chem. Soc. 2014, 136, 5828. (e) Bonet, A.; Odachowski, M.; Leonori, D.; Essafi, S.; Aggarwal, V. K. Nat. Chem. 2014, 6, 584.

(2) (a) Xi, Y.; Hartwig, J. F. J. Am. Chem. Soc. 2016, 138, 6703. (b) Ceinos, M. G.; Parra, A.; Heras, V. M.; Tortosa, M. Angew. Chem., Int. Ed. 2016, 55, 6969. (c) Sasaki, Y.; Zhong, C.; Sawamura, M.; Ito, H. J. Am. Chem. Soc. 2010, 132, 1226.

(3) (a) Zhang, H.; Lu, Z. ACS Catal. 2016, 6, 6596. (b) Zhang, L.; Zuo, Z.; Wan, Z.; Huang, Z. J. Am. Chem. Soc. 2014, 136, 15501. (c) He, Z.-T.; Zhao, Y.-S.; Tian, P.; Wang, C.-C.; Dong, H.-Q.; Lin, G.-Q. Org. Lett. 2014, 16, 1426. (d) Feng, X.; Jeon, H.; Yun, J. Angew. Chem., Int. Ed. 2013, 52, 3989. (e) Mazet, C.; Gérard, D. Chem. Commun. 2011, 47, 298. (f) Corberán, R.; Mszar, N. W.; Hoveyda, A. H. Angew. Chem., Int. Ed. 2011, 50, 7079.

(4) For recent partial summary of other ways, including stereoconvergent cross-coupling methods to prepare chiral organoboron derivatives, see: Schmidt, J.; Choi, J.; Liu, A. T.; Slusarczyk, M.; Fu, G. C. Science 2016, 354, 1265.

(5) For other approaches to chiral tertiary boronic esters, see: (a) Hu, N.; Zhao, G.; Zhang, Y.; Liu, X.; Li, G.; Tang, W. J. Am. Chem. Soc. 2015, 137, 6746. (b) Hong, K.; Liu, X.; Morken, J. P. J. Am. Chem. Soc. 2014, 136, 10581. (c) Pulis, A. P.; Blair, D. J.; Torres, E.; Aggarwal, V. K. J. Am. Chem. Soc. 2013, 135, 16054. (d) O'Brien, J. M.; Lee, K. S.; Hoveyda, A. H. J. Am. Chem. Soc. 2010, 132, 10630. (e) Chen, I. H.; Yin, L.; Itano, W.; Kanai, M.; Shibasaki, M. J. Am. Chem. Soc. 2009, 131, 11664.

(6) (a) Shoba, V. M.; Thacker, N. C.; Bochat, A. J.; Takacs, J. M. Angew. Chem., Int. Ed. 2016, 55, 1465. (b) Hoang, G. L.; Yang, Z. D.; Smith, S. M.; Pal, R.; Miska, J. L.; Perez, D. E.; Pelter, L. S. W.; Zeng, X. C.; Takacs, J. M. Org. Lett. 2015, 17, 940. (c) Smith, S. M.; Takacs, J. M. J. Am. Chem. Soc. 2010, 132, 1740. (d) Smith, S. M.; Thacker, N. C.; Takacs, J. M. J. Am. Chem. Soc. 2008, 130, 3734.

(7) (a) Chary, B. C.; Kim, S. Org. Biomol. Chem. 2013, 11, 6879. (b) Zhao, D.; Nimphius, C.; Lindale, M.; Glorius, F. Org. Lett. 2013, 15, 4504. (c) Bera, M.; Sahoo, S. K.; Maiti, D. ACS Catal. 2016, 6, 3575 .
(8) Yields for phosphonate-directed CAHB are typically slightly higher (ca. 4-5\%) using T2 than those obtained using T1; the er of 4a obtained using $\mathbf{T} 1$ is $98.5: 1.5$.

(9) (a) Kaplan, A. P.; Bartlett, P. A. Biochemistry 1991, 30, 8165. (b) Tebby, J. C. Aminophosphonic and aminophosphinic acids: chemistry and biological activity; Kukhar, V. P., Hudson, H. R., Eds.; John Wiley \& Sons Ltd: Chichester, 2000. (c) Horsman, G. P.; Zechel, D. L. Chem. Rev. Article ASAP, DOI: 10.1021/acs.chemrev.6b00536.

(10) For a review of synthetic methods to construct $\alpha$ - and $\beta$ aminophosphonates, see: Ma, J. A. Chem. Soc. Rev. 2006, 35, 630.

(11) (a) Yan, Z.; Wu, B.; Gao, X.; Chen, M.-W.; Zhou, Y.-G. Org. Lett. 2016, 18, 692. (b) Corbett, M. T.; Johnson, J. S. J. Am. Chem. Soc. 2013, 135, 594. (c) Zhang, J.; Li, Y.; Wang, Z.; Ding, K. Angew. Chem., Int. Ed. 2011, 50, 11743.

(12) Bueschleb, M.; Dorich, S.; Hanessian, S.; Tao, D.; Schenthal, K. B.; Overman, L. E. Angew. Chem., Int. Ed. 2016, 55, 4156 and references cited therein.

(13) Sonawane, R. P.; Jheengut, V.; Rabalakos, C.; LaroucheGauthier, R.; Scott, H. K.; Aggarwal, V. K. Angew. Chem., Int. Ed. 2011, 50, 3760.

(14) Hon, Y.-S.; Lin, S. W.; Lu, L.; Chen, Y.-J. Tetrahedron 1995, 51, 5019.

(15) Chatterjee, B.; Gunanathan, C. Chem. Commun. 2014, 50, 888.

(16) Abdel-Magid, A. F.; Carson, K. G.; Harris, B. D.; Maryanoff, C. A.; Shah, R. D. J. Org. Chem. 1996, 61, 3849.

(17) (a) Mlynarski, S. N.; Karns, A. S.; Morken, J. P. J. Am. Chem. Soc. 2012, 134, 16449. (b) Bagutski, V.; Elford, T. G.; Aggarwal, V. K Angew. Chem., Int. Ed. 2011, 50, 1080.

(18) (a) Patel, H. H.; Sigman, M. S. J. Am. Chem. Soc. 2016, 138 14226. For synthesis of $\alpha$-amino phosphonates via Curtis rearrangement, see: (b) Moore, J. D.; Sprott, K. T.; Hanson, P. R. J. Org. Chem. 2002, 67, 8123.

(19) Alkene reduction is a significant side reaction for (Z)-3a.

(20) Majeed, R.; Reddy, M. V.; Chinthakindi, P. K.; Sangwan, P. L.; Hamid, A.; Chashoo, G.; Saxena, A. K.; Koul, S. Eur. J. Med. Chem. 2012, 49, 55.

(21) For recent syntheses of bakuchiol, see: (a) Xiong, Y.; Zhang, G. Org. Lett. 2016, 18, 5094. (b) Esumi, T.; Yamamoto, C.; Fukuyama, Y. Synlett 2013, 24, 1845. (c) Gao, F.; McGrath, K. P.; Lee, Y.; Hoveyda, A. H. J. Am. Chem. Soc. 2010, 132, 14315.

(22) Reichwein, J. F.; Pagenkopf, B. L. J. Am. Chem. Soc. 2003, 125, 1821.

(23) Corey, E. J.; Kwiatkowski, G. T. J. Am. Chem. Soc. 1966, 88, 5654.

(24) Yamagishi, T.; Muronoi, S.; Hikishima, S.; Shimeno, H.; Soeda, S.; Yokomatsu, T. J. Org. Chem. 2009, 74, 6350 and references cited therein.

(25) The stereochemistry of CAHB is assigned based on correlation via this synthesis.

(26) Guillaneux, D.; Zhao, S.-H.; Samuel, O.; Rainford, D.; Kagan, K. H. J. Am. Chem. Soc. 1994, 116, 9430. 